\title{
Expression and clinical significance of androgen receptor in bladder cancer: A meta-analysis
}

\author{
JINBO CHEN ${ }^{1,2^{*}}$, YU CUI $^{*}$, PENG LI ${ }^{3}$, LONGFEI LIU ${ }^{1}, \mathrm{CHAO} \mathrm{LI}^{1}$ and XIONGBING ZU ${ }^{1}$ \\ ${ }^{1}$ Department of Urology, Xiangya Hospital, Central South University, Changsha, Hunan 410008, \\ P.R. China; ${ }^{2}$ Department of Pathology and Laboratory Medicine, The Wilmot Cancer Center, University of \\ Rochester Medical Center, Rochester, NY 14620, USA; ${ }^{3}$ Minimally Invasive Urology Center, Shandong \\ Provincial Hospital, Shandong University School of Medicine, Jinan, Shandong 250021, P.R. China
}

Received March 27, 2017; Accepted June 28, 2017

DOI: $10.3892 /$ mco.2017.1389

\begin{abstract}
Emerging evidence has demonstrated that androgen receptor (AR) is a promising therapeutic target for bladder cancer. However, the relationship between AR expression and its clinical significance remains controversial. The present in-depth meta-analysis aimed to investigate the correlation between AR expression and clinicopathological features, as well as prognostic value in bladder cancer. A systematic search was performed from PubMed, Web of Knowledge, Embase and the Cochrane Central Search Library by January 2017. The correlation between AR expression and tumor stage, tumor grade, recurrence free survival and progression free survival for patients with bladder cancer was evaluated. A total of 12 relevant studies with 1,652 patient samples were included. AR expression positively correlated with low tumor grade [odds ratio (OR), 1.95; 95\% confidence interval (CI), 1.36-2.81], low tumor stage (OR, 2.06; 95\% CI, 1.02-4.16) and low recurrence rate [hazard ratio (HR), 0.48; 95\% CI, 0.31-0.75] in Caucasian patients. While, its expression had no significant impact on cancer susceptibility (OR, 1.62; 95\% CI, 0.19-13.72; $\mathrm{P}=0.44)$ and progression-free survival (HR, 1.20; 95\% CI, 0.86-1.66; $\mathrm{P}=0.77$ ). The present meta-analysis indicated that AR expression correlates with tumor grade, clinical stage and recurrence rates in the specified population and classification system.
\end{abstract}

Correspondence to: Professor Xiongbing $\mathrm{Zu}$, Department of Urology, Xiangya Hospital, Central South University, 87 Xiang Ya Road, Changsha, Hunan 410008, P.R. China

E-mail:whzuxb@163.com

${ }^{*}$ Contributed equally

Abbreviations: AR, androgen receptor; RFS, recurrence-free survival; PFS, progression-free survival; OS, overall survival; OR, odds ratio; HR, hazard ratio; $\mathrm{CI}$, confidence interval; IHC, immunohistochemistry; NI, non-invasive; MI, muscle invasive

Key words: bladder cancer, androgen receptor, meta-analysis, prognosis
Further studies are required to determine the precise role of $\mathrm{AR}$ in bladder cancer.

\section{Introduction}

Bladder cancer is one of the most common types of urinary tract malignancy worldwide (1). It is clinically characterized by its progression, recurrence, metastasis and drug resistance (2). Notably, due to the lifetime requirement for monitoring tumor recurrence, the typical cost of bladder cancer from diagnosis to mortality is the highest among all cancer types (3). To better understand the molecular mechanisms of the disease, research is under way so that novel treatments may be identified. However, therapeutic methods have remained essentially unchanged over the past three decades, indicating an urgent need to further research this malignancy (4).

Interestingly, it has been reported that the incidence of bladder cancer is 3-4 times greater in men than in women (5). Lifestyle or environmental factors, such as cigarette smoke and industrial chemicals, are believed to be responsible for the gender-specific disparity in bladder cancer morbidity and aggressiveness (6). However, it remains a preferential disease in men even after controlling for these carcinogens (6). Thus, a hypothesis has been proposed that the androgen receptor (AR) and related signaling pathways are involved in the etiology and progression of bladder cancer (7).

AR signals have been demonstrated to correlate with bladder cancer development and progression both in vitro and in vivo (7). However, the correlation between AR expression and its clinical significance remains controversial in patients with bladder cancer. Some evidence has indicated that AR expression is related to bladder cancer pathology grade, clinical stage or prognosis (8-10). While a multi-institutional study revealed no correlations (11). Thus, the present meta-analysis aimed to evaluate the expression and clinical significance of AR in bladder cancer. To the best of our knowledge, this is the first meta-analysis to investigate the impact of AR expression on bladder cancer.

\section{Data collection methods}

Publication search strategy. In accordance with the Preferred Reporting Items for Systematic Reviews and Meta-analyses 
guidelines (12), a systematic review of literature was performed in January 2017 using PubMed (ncbi.nlm.nih.gov/pubmed), Web of Knowledge (webofknowledge.com), Embase (embase. com) and the Cochrane Central Search Library (cochranelibrary.com). Search terms used included 'androgen receptor,' 'AR,' 'bladder,' 'cancer,' 'carcinoma' and 'tumor.' All abstracts and review articles on this topic were reviewed, and references of original studies were identified by manual search.

Inclusion criteria and exclusion criteria. Eligible studies had to meet the following selection criteria: i) Studies had to evaluate the association between AR and bladder cancer; ii) the report contained key information about AR expression and bladder cancer susceptibility, tumor grade, lymph node metastasis recurrence-free survival (RFS) or progression-free survival (PFS); iii) studies published in English; and iv) conference abstracts, reviews and letters to editors were not included. Studies with overlapping or insufficient data were excluded.

Data extraction. Two independent reviewers (Jinbo Chen and $\mathrm{Yu}$ Cui) extracted the information from eligible studies to the inclusion criteria. Disagreement was resolved during a consensus with a third reviewer (Xiongbing $\mathrm{Zu}$ ). The literature data and demographic were extracted individually. Odds ratio (OR) and 95\% confidence intervals (CI) were used to estimate the association between AR expression and bladder cancer susceptibility, tumor grade and clinical stage. Hazard ratio (HR) and its 95\% CIs were used to elevate the association between AR expression and RFS and PFS. If available, the HRs with their 95\% CIs and P-values were collected from the original article. If not, HRs and their 95\% CIs were calculated using the data of observed cancer progression/recurrences, the data of samples in each group or the data provided by the authors. If only survival curves were available, data was extracted from the graphical survival plots and the HRs were estimated $(13,14)$. The quality of studies was evaluated using the Newcastle-Ottawa Scale (15). Scores of 7-9 were defined as high quality study, and a score $<7$ as low quality study.

Statistical analysis. A meta-analysis was performed to assess the association between AR expression and bladder cancer susceptibility, tumor grade, tumor stage, RFS as well as PFS. Statistical heterogeneity was assessed using a formal Q-statistic as well as I-squared, with the statistical significance level set at 0.05 (16). A fixed-effects model was used when no heterogeneity was found; otherwise, the random-effects model was used to calculate pooled ORs. Publication bias was evaluated by Egger's (17) and Begg's (18) test. $\mathrm{P}<0.05$ was considered to indicate a statistically significant difference. All statistical analyses were implemented in STATA 11.0 statistical software (StataCorp LP, College Station, TX, USA).

\section{Results}

Literature search. A total of 235 studies were identified from the database or manual search. According to the selection criteria, 223 studies were excluded, resulting in 12 studies with 1,652 patients for analysis. A flow chart of article selection summarizes the main characteristics of included studies, as demonstrated in Fig. 1. Finally, data were available from five

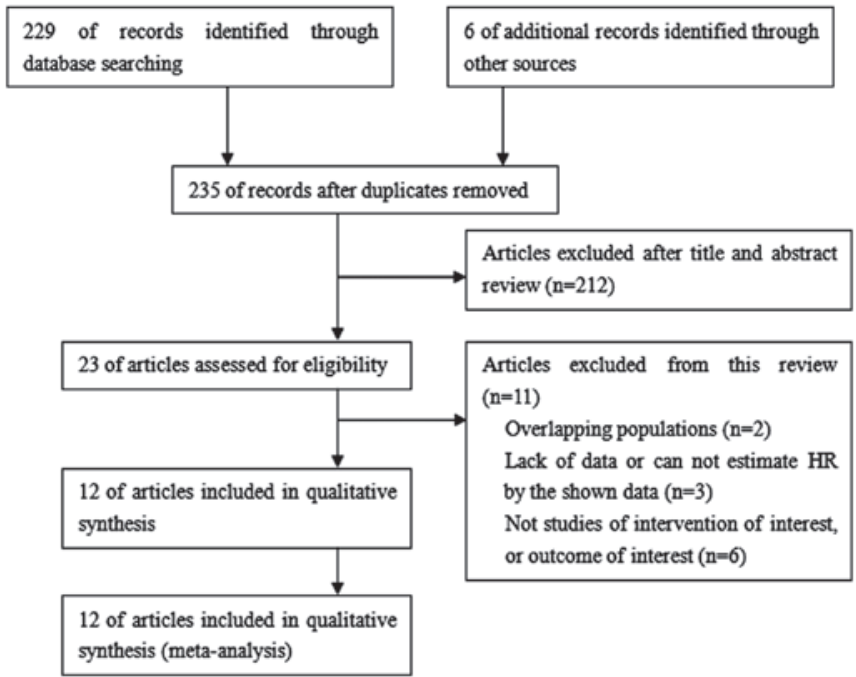

Figure 1. Flow chart of articles reviewed and included in the present meta-analysis.

studies on AR expression and bladder cancer susceptibility (568 tumor cases vs. 523 normal controls), six studies on AR expression and tumor grade ( 387 low grade vs. 663 high grade cases), nine studies on AR expression and tumor stage (582 non-invasive vs. 712 invasive cases), five studies on AR expression and RFS (414 cases) and four studies on AR expression and PFS (319 cases). The essential information of the included studies was listed in Table I (8-11,19-26).

$A R$ expression and bladder cancer susceptibility. In total, five studies reported AR expression and bladder cancer susceptibility, involving 1,091 samples (568 urothelial tumor vs. 523 normal urothelium tissues). There was statistical heterogeneity between these trials (I-squared=94.2\%; $\mathrm{P}<0.001$ ), so a random-effects model was used in the analysis. The reason for this may be that AR positive and negative cases in the control group of these literatures were different. The district and ethnicity differences may contribute to this heterogeneity, and so subgroup analysis was conducted according to ethnicity. Overall, no significant association was observed between AR expression and bladder cancer susceptibility (OR, 1.62; 95\% CI, 0.19-13.72; P=0.44; Fig. 2). Subgroup analysis by different ethnicity demonstrated that AR expression had no significant association with bladder cancer susceptibility both for Caucasian (OR, 1.13; 95\% CI, 0.05-24.47; $\mathrm{P}=0.98)$ and Asian patients (OR, 4.34; 95\% CI, 0.01-3544.09; $\mathrm{P}=0.66$; Table II).

AR expression and tumor grade. A total of six studies investigated the relationship between AR expression and bladder cancer grade, including 1,050 cases. No statistical heterogeneity between trials was identified (I-squared $=43.9 \%$; $\mathrm{P}=0.113$ ), so a fixed-effects model was used in the analysis. Overall, the AR expression was positively correlated with low bladder cancer grade (OR, 1.74; 95\% CI, 1.27-2.37; $\mathrm{P}<0.01$; Fig. 3). Subgroup analysis by different ethnicities demonstrated that AR expression correlated with low bladder cancer grade for Caucasian patients (OR, 1.95; 95\% CI, 1.36-2.81; $\mathrm{P}<0.01)$. However, such association was not 


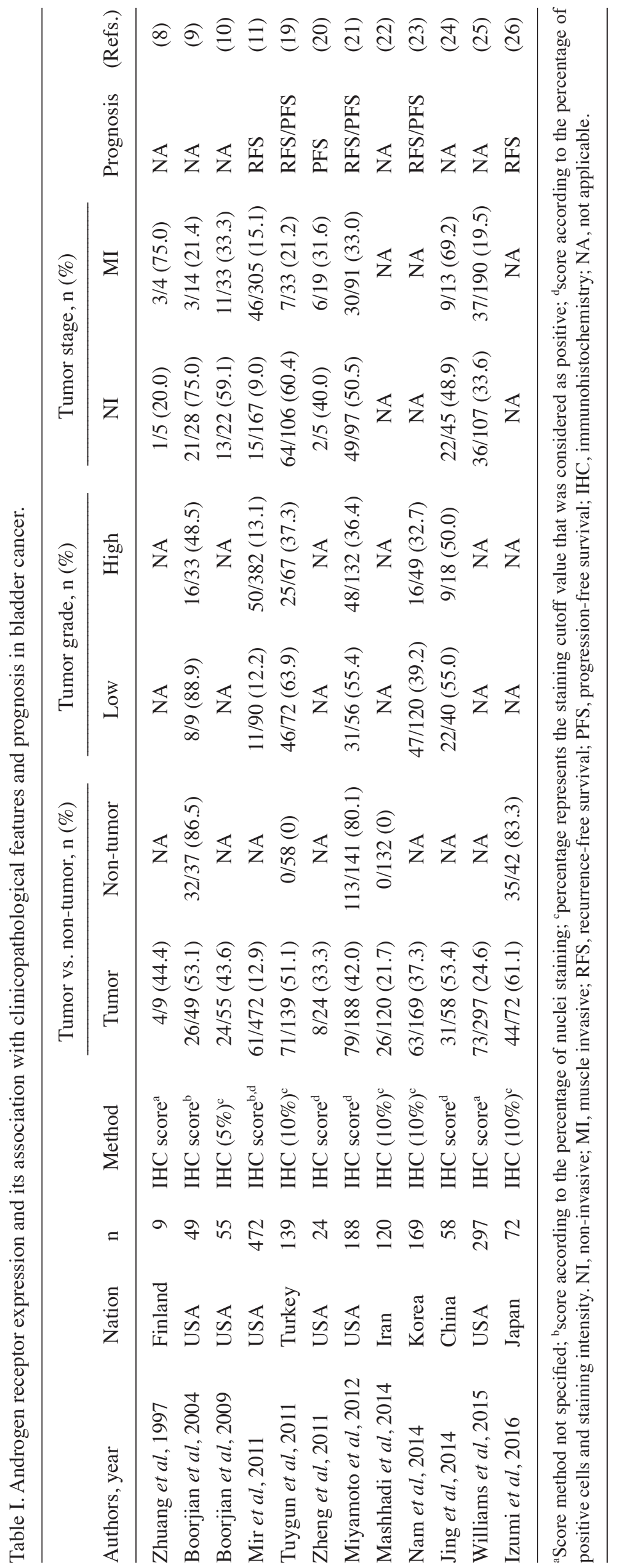


Table II. Subgroup analysis for androgen receptor and clinicopathological features and prognosis in bladder cancer.

\begin{tabular}{|c|c|c|c|c|c|c|}
\hline \multirow[b]{2}{*}{ Category } & \multirow[b]{2}{*}{$\mathrm{n}$} & \multirow[b]{2}{*}{ Odds ratio } & \multirow[b]{2}{*}{ 95\% confidence interval } & \multirow[b]{2}{*}{ P-value } & \multicolumn{2}{|c|}{ Heterogeneity } \\
\hline & & & & & I-squared, \% & P-value \\
\hline \multicolumn{7}{|c|}{ Susceptibility due to ethnicity } \\
\hline Caucasian & 3 & 1.13 & $0.05-24.47$ & 0.98 & 95.30 & $<0.01$ \\
\hline Asian & 2 & 4.34 & $0.01-3544.09$ & 0.66 & 95.10 & $<0.01$ \\
\hline \multicolumn{7}{|c|}{ Tumor grade and ethnicity } \\
\hline Caucasian & 4 & 1.95 & $1.36-2.81$ & $<0.01$ & 60.90 & 0.05 \\
\hline Asian & 2 & 1.30 & $0.72-2.35$ & 0.39 & 0 & 0.9 \\
\hline \multicolumn{7}{|c|}{ Classification system } \\
\hline $2004 \mathrm{WHO}$ & 4 & 1.94 & $1.35-2.80$ & $<0.01$ & 11 & 0.34 \\
\hline $1998 \mathrm{WHO}$ & 2 & 1.25 & $0.68-2.32$ & 0.47 & 72.80 & 0.05 \\
\hline \multicolumn{7}{|c|}{ Tumor stage and ethnicity } \\
\hline Caucasian & 8 & 2.06 & $1.02-4.16$ & 0.04 & 77 & $<0.01$ \\
\hline Asian & 1 & 0.43 & $0.11-1.58$ & 0.20 & NA & NA \\
\hline \multicolumn{7}{|c|}{ Recurrence-free survival and ethnicity } \\
\hline Caucasian & 3 & $0.48^{\mathrm{a}}$ & $0.31-0.75$ & 0.01 & 0 & 0.42 \\
\hline Asian & 2 & $1.13^{\mathrm{a}}$ & $0.87-1.47$ & 0.74 & 47.70 & 0.125 \\
\hline \multicolumn{7}{|c|}{ Progression-free survival and ethnicity } \\
\hline Caucasian & 3 & $1.31^{\mathrm{a}}$ & $0.92-1.85$ & 0.13 & 55 & 0.07 \\
\hline Asian & 1 & $0.61^{\mathrm{a}}$ & $0.23-1.62$ & 0.32 & NA & NA \\
\hline
\end{tabular}

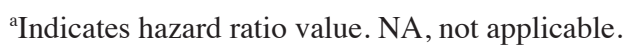

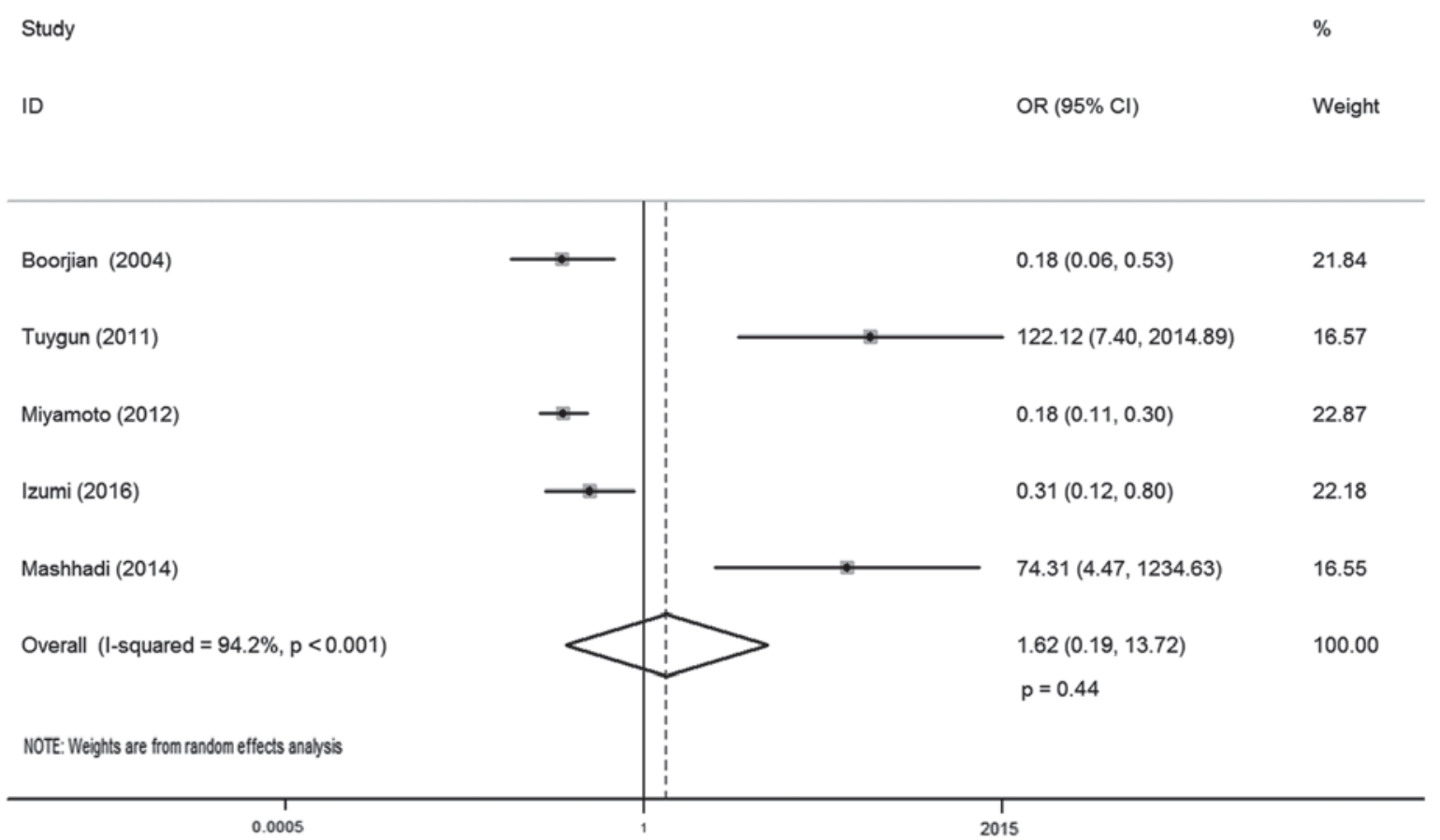

Figure 2. Forest plot of OR for bladder cancer susceptibility. Square indicates point estimate of each study. Size of square indicates relative contribution of each study. Solid horizontal line represents $95 \%$ CI of each study. The diamond indicates the pooled OR value. OR, odds ratio; CI, confidence interval.

observed for Asian populations (OR, 1.30; 95\% CI, 0.72-2.35; $\mathrm{P}=0.39$ ). Subgroup analysis by different pathology classification systems demonstrated that AR expression correlated with low bladder cancer grade for the 2004 World Health Organization (WHO) classification system (OR, 1.94; 95\% CI, 1.35-2.80; $\mathrm{P}<0.01)$. However, such relationship was not 


\begin{tabular}{|c|c|c|}
\hline Boorjian (2004) & $8.50(0.95,75.80)$ & 1.29 \\
\hline Mir (2010) & $0.92(0.46,1.86)$ & 28.36 \\
\hline Nam (2014) & $1.33(0.66,2.68)$ & 23.42 \\
\hline Tuygun (2011) & $2.97(1.49,5.93)$ & 15.84 \\
\hline Miyamoto (2012) & $2.17(1.15,4.10)$ & 21.63 \\
\hline Jing (2014) & $1.22(0.40,3.72)$ & 9.46 \\
\hline \multirow[t]{2}{*}{ Overall $(\mathrm{I}-$ squared $=43.9 \%, p=0.113$ ) } & $1.74(1.27,2.37)$ & 100.00 \\
\hline & $p<0.01$ & \\
\hline $\begin{array}{c}1 \\
0.0132\end{array}$ & 5.8 & \\
\hline
\end{tabular}

Figure 3. Forest plot of OR for tumor grade. Square indicates point estimate of each study. Size of square indicates relative contribution of each study. The horizontal line crossing the square represents the $95 \%$ CI. The diamond indicates the pooled OR value. OR, odds ratio; CI, confidence interval.

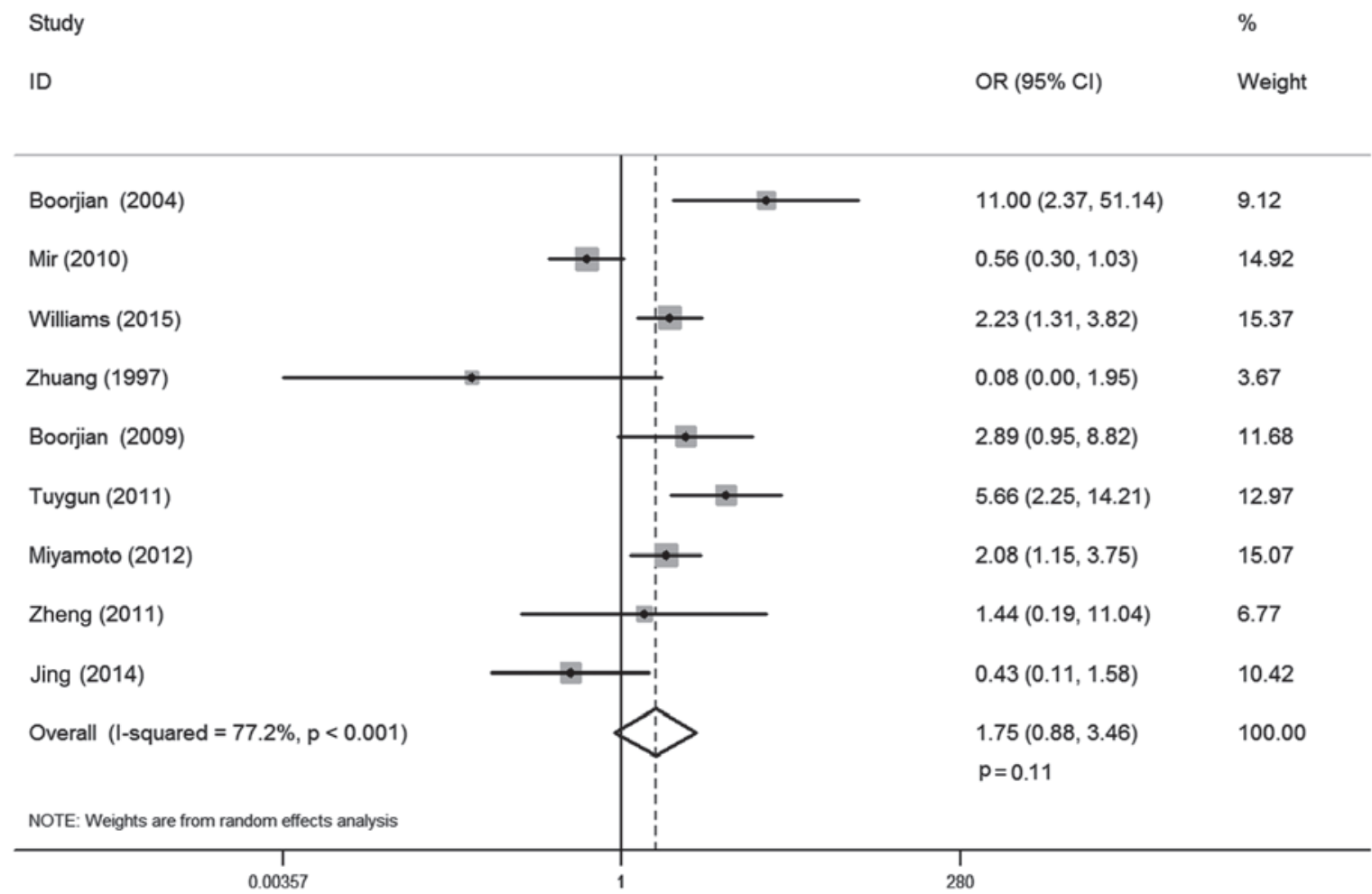

Figure 4. Forest plot of OR for tumor stage. Square indicates point estimate of each study. Size of square indicates relative contribution of each study. Solid horizontal line represents $95 \% \mathrm{CI}$ of each study. The diamond indicates the pooled OR value. OR, odds ratio; CI, confidence interval.

found for the 1998 WHO classification system (OR, 1.25; 95\% CI, 0.68-2.32; P=0.47; Table II).

AR expression and tumor stage. There were 9 studies that reported the correlation between AR expression and bladder cancer stage, involving 1,294 cases. Statistical heterogeneity was observed between trials (I-squared $=77.2 \% ; \mathrm{P}<0.001$ ). Thus, a random-effects model was used in the analysis. The district and ethnicity differences may contribute to this heterogeneity, so subgroup analysis according to ethnicity was conducted. 
Study

ID
$\operatorname{HR}(95 \% \mathrm{Cl}) \quad$ Weight

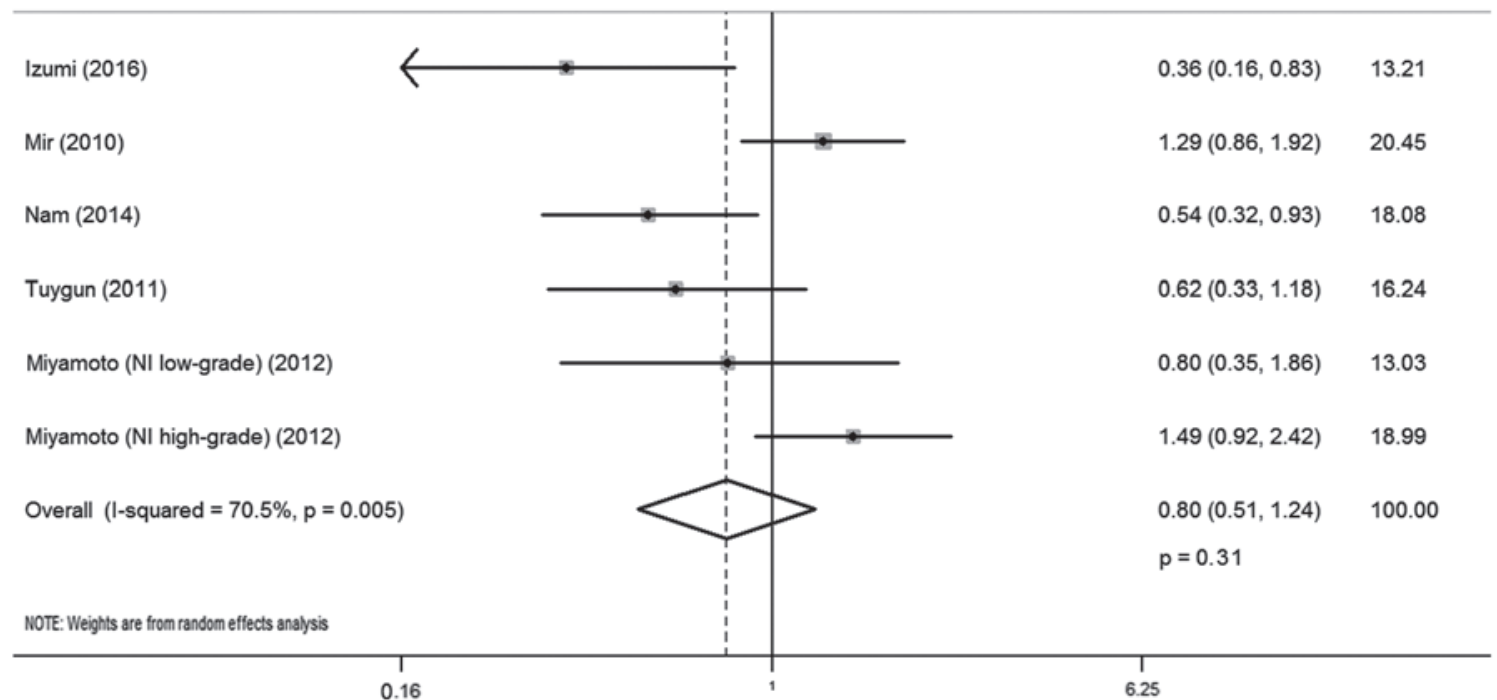

Figure 5. Forest plot of HR for recurrence-free survival. Square indicates point estimate of each study. Size of square indicates relative contribution of each study. The horizontal line crossing the square represents the 95\% CI. The diamond indicates the pooled HR value. NI, non-invasive; MI, muscle invasive; HR, hazard ratio; CI, confidence interval.

Study

ID
$\%$

$\mathrm{HR}(95 \% \mathrm{Cl}) \quad$ Weight

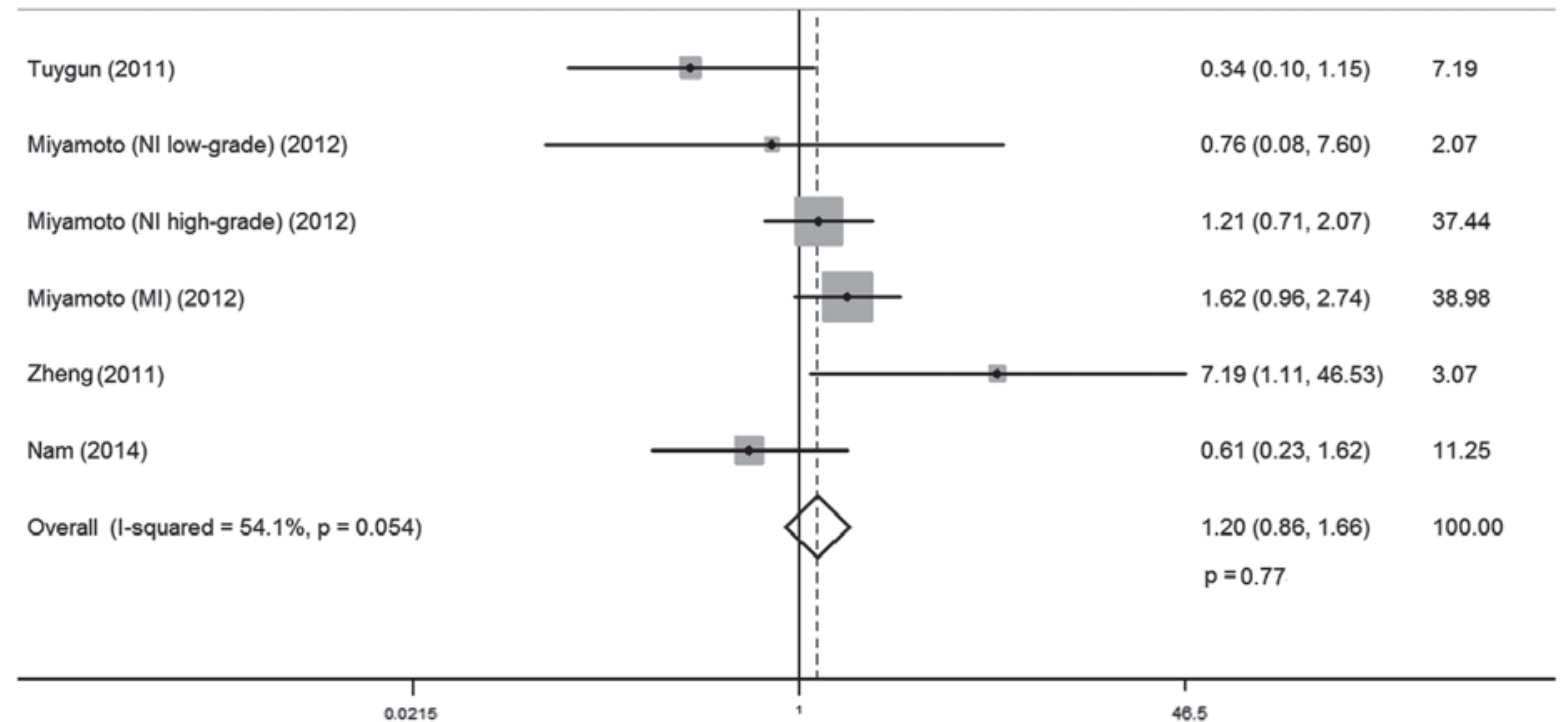

Figure 6. Forest plot of HR for progression-free survival. Square indicates point estimate of each study. Size of square indicates relative contribution of each study. The horizontal line crossing the square represents the 95\% CI. The diamond indicates the pooled HR value. NI, non-invasive; MI, muscle invasive; HR, hazard ratio; $\mathrm{CI}$, confidence interval.

Overall, no significant association was demonstrated between AR expression and tumor stage (OR, 1.75; 95\% CI, 0.88-3.46; $\mathrm{P}=0.11$; Fig. 4). Subgroup analysis by ethnicity demonstrated that AR expression was positively correlated with non-invasive tumors compared with muscle invasive stage for Caucasian patients (OR, 2.06; 95\% CI, 1.02-4.16; $\mathrm{P}=0.04)$, whereas such association was not found in Asian populations (OR, 0.43; 95\% CI, 0.11-1.58; $\mathrm{P}=0.20$; Table II).

AR expression and RFS. A total of five studies reported the correlation between AR expression and RFS, involving 414 cases. There was statistical heterogeneity between trials 


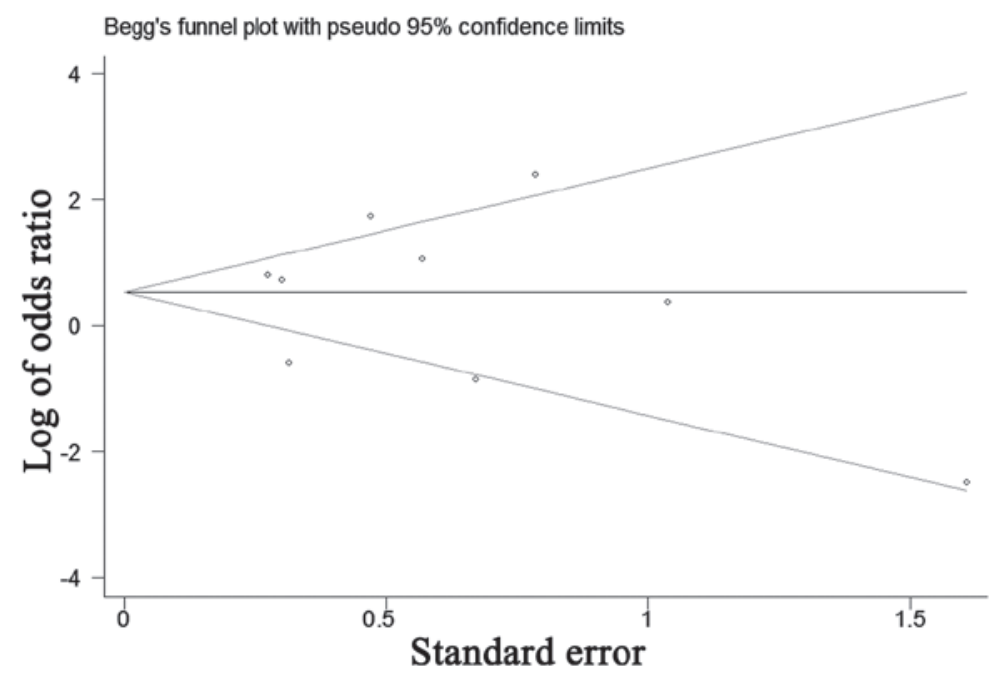

Figure 7. Funnel plot for potential publication bias of tumor stage.

(I-squared $=70.5 \% ; \mathrm{P}=0.005$ ), and so a random-effects model was used in the analysis. The district and ethnicity differences as well as the limited number of patients included in the studies may have contributed to this heterogeneity; therefore, subgroup analysis according to ethnicity was conducted. Overall, no significant association was observed between AR expression and RFS (HR, 0.80; 95\% CI, 0.51-1.24; $\mathrm{P}=0.31$; Fig. 5). Subgroup analysis by ethnicity indicated that AR expression was correlated with lower recurrence rate for Caucasian patients with bladder cancer (HR, 0.48; 95\% CI, 0.31-0.75; $\mathrm{P}=0.01)$, whereas such association was not observed for Asian patients (HR, 1.13; 95\% CI, 0.87-1.47; $\mathrm{P}=0.74$ ).

AR expression and PFS. A total of four studies investigated the relationship between AR expression and PFS, involving 319 cases. There was no statistical heterogeneity between trials (I-squared=54.1\%; $\mathrm{P}=0.054$ ), so a fixed-effects model was used in the analysis. Overall, no significant association was observed between AR expression and PFS (HR, 1.20; 95\% CI, 0.86-1.66; P=0.77; Fig. 6) Subgroup analysis by ethnicity demonstrated that AR expression was not correlated with progression free rates for Caucasian and Asian patients with bladder cancer (HR, 1.31; 95\% CI, 0.92-1.85; $\mathrm{P}=0.13$; and HR, 0.61; 95\% CI, 0.23-1.62; $\mathrm{P}=0.32$, respectively; Table II).

Publication bias. The funnel plot for the relationship between AR expression and tumor stage was demonstrated in Fig. 7. P-values for Begg's adjusted rank correlation test was 0.75 and the Egger's regression asymmetry test was 0.48 . The results did not reveal any evidence of publication bias.

\section{Discussion}

The AR gene, located in the $\mathrm{X}$ chromosome (q11-12), is a member of the nuclear receptor superfamily (27). As a transcription factor, it mediates physiological activities by binding to androgen. It has been demonstrated that androgen signaling has been linked to regulation of proliferation, motility and cell death in urinary malignancies $(28,29)$. Importantly, it has essential roles in malignancies of the prostate, bladder, kidney, lung, breast and liver (30-32). To date, aiming to investigate the possible role of AR in bladder cancer, various studies have defined AR expression patterns and functions in these patients' samples (8-11). However, due to diversity in sample sizes, ethnicity, immunohistochemistry (IHC) techniques, scoring systems and interventions, the results remain inconsistent. Therefore, the present in-depth meta-analysis aimed to reveal the correlation between AR expression and clinicopathological features, as well as prognosis in patients with bladder cancer.

Notable efforts have been made to study AR expression in bladder cancer tissues compared with normal bladder samples. However, there is large discrepancy among the results of these studies. In the present meta-analysis, the AR positive rate ranged from $12.9-61.1 \%$ in bladder cancer tissues, while this rate ranged from $0-86.5 \%$ in normal bladder urothelium. Studies by Tuygun et al (19) and Mashhadi et al (22) revealed that AR expression was much higher in bladder cancer tissues, compared with $0 \%$ positivity in normal tissues. However, data from studies by Boorjian et al (9), Miyamoto et al (21) and Izumi et al (26) demonstrated that the AR positive rate was lower in bladder cancer tissues than in normal bladder samples. The present meta-analysis with five studies and 1,091 samples demonstrated no significant association between AR expression and bladder cancer susceptibility.

Since cancer biomarker expression may increase in the early stage and decrease in later periods, AR expression in different tumor grades and stages was further investigated in the present meta-analysis. Unexpectedly, the correlation results between AR expression and bladder cancer development have not reached a consensus. Tuygun et al (19) demonstrated that AR expression negatively correlated with tumor grade and clinical stage in 139 patients. Also, this relationship was indicated by Miyamoto et al (21). In contrast, a multi-institutional study by Mir et al (11) reported that AR expression was not associated with pathological grade and stage in bladder cancer. Concluding these data, the present subgroup meta-analysis indicated that AR expression was positively correlated with low tumor grade and non-invasive tumor stage for Caucasian patients, while significant associations for tumor grade and stage were not observed in Asian patients. 
Furthermore, the pathological grading system of bladder cancer was updated in 2004 (33). Subgroup analysis based on this new classification system in the present meta-analysis demonstrated that AR expression had a positive correlation with low bladder cancer grade. Similarly, subgroup analysis indicated that AR expression was positively correlated with non-muscle invasive tumors, compared with muscle invasive tumors in the Caucasian population. These results revealed that AR expression decreased with increasing grade and stage, indicating that low AR expression was associated with bladder cancer development in the specified population and classification system.

Bladder cancer is a disease of high recurrence and easy progression (6). Thus, it is necessary to monitor tumor recurrence throughout a patient's lifetime (34). Unfortunately, $\sim 70 \%$ of patients presenting with superficial bladder tumor develop recurrence and $10-20 \%$ of these patients progress to muscle invasive bladder cancer (35). Recently, the controversial relationship between AR expression and bladder cancer prognosis has been reported. Studies by Nam et al (23) and Izumi et al (26) indicated that AR-positive patients had a significantly lower risk of tumor recurrence compared with those with AR-negative tumors. Contrastingly, Mir et al (11) demonstrated that loss of AR expression was not associated with clinical outcome, including RFS. Interestingly, Miyamoto et al (21) divided bladder patients into non-invasive low grade, non-invasive high grade and muscle invasive groups, and analyzed AR expression and RFS and PFS in these groups. The present subgroup analysis demonstrated that AR expression was positively correlated with lower recurrence rates in Caucasian patients with bladder cancer; however, its expression had no significant impact on PFS. As cases included in the present analysis were limited, more studies are required to clarify AR expression and its prognostic role in bladder cancer in the future.

Several limitations should be noted in the present meta-analysis. First, all data included were from retrospective studies. This may potentially enlarge certain bias, such as selection bias. Second, case quantities in some studies were confined, particularly for the Asian population. Third, publication on AR expression and prognosis of overall survival was not sufficient, which restrained our ability to conduct analysis. Forth, the standard for IHC positive staining between the studies was not consistent, which may cause some heterogeneity. Furthermore, several HRs for RFS and PFS were calculated based on the data extracted from the survival curve, and this may also induce some errors.

In conclusion, the present study provided evidence for correlations between AR expression and clinicopathological features, as well as prognosis in patients with bladder cancer. Although heterogeneity exists in the included studies, the present meta-analysis demonstrated that AR expression was correlated with tumor grade, clinical stage and recurrence rates in the specified population and classification system. No association was observed between AR and bladder cancer susceptibility or PFS. Accordingly, further mechanistic studies are required to determine the precise functional role of $A R$ signaling in the development and progression of bladder cancer.

\section{Acknowledgments}

The present study was supported by the National Natural Science Foundation of China (grant no. 81572523) and the Fundamental Research Funds for the Central Universities of Central South University (grant no. 2016zzts121).

\section{References}

1. Siegel RL, Miller KD and Jemal A: Cancer statistics, 2015. CA Cancer J Clin 65: 5-29, 2015.

2. Herr HW: Transurethral resection of muscle-invasive bladder cancer: 10-year outcome. J Clin Oncol 19: 89-93, 2001.

3. Sievert KD, Amend B, Nagele U, Schilling D, Bedke J, Horstmann M, Hennenlotter J, Kruck S and Stenzl A: Economic aspects of bladder cancer: What are the benefits and costs? World J Urol 27: 295-300, 2009.

4. Carneiro BA, Meeks JJ, Kuzel TM, Scaranti M, Abdulkadir SA and Giles FJ: Emerging therapeutic targets in bladder cancer. Cancer Treat Rev 41: 170-178, 2015.

5. Dobruch J, Daneshmand S, Fisch M, Lotan Y, Noon AP, Resnick MJ, Shariat SF, Zlotta AR and Boorjian SA: Gender and bladder cancer: A collaborative review of etiology, biology, and outcomes. Eur Urol 69: 300-310, 2016.

6. Kamat AM, Hahn NM, Efstathiou JA, Lerner SP, Malmström PU, Choi W, Guo CC, Lotan Y and Kassouf W: Bladder cancer. Lancet 388: 2796-2810, 2016.

7. Li Y,Izumi K and Miyamoto $\mathrm{H}$ : The role of the androgen receptor in the development and progression of bladder cancer. Jpn J Clin Oncol 42: 569-577, 2012.

8. Zhuang YH, Bläuer M, Tammela $\mathrm{T}$ and Tuohimaa P: Immunodetection of androgen receptor in human urinary bladder cancer. Histopathology 30: 556-562, 1997.

9. Boorjian S, Ugras S, Mongan NP, Gudas LJ, You X, Tickoo SK and Scherr DS: Androgen receptor expression is inversely correlated with pathologic tumor stage in bladder cancer. Urology 64: 383-388, 2004.

10. Boorjian SA, Heemers HV, Frank I, Farmer SA, Schmidt LJ, Sebo TJ and Tindall DJ: Expression and significance of androgen receptor coactivators in urothelial carcinoma of the bladder. Endocr Relat Cancer 16: 123-137, 2009.

11. Mir C, Shariat SF, van der Kwast TH, Ashfaq R, Lotan Y, Evans A, Skeldon S, Hanna S, Vajpeyi R, Kuk C, et al: Loss of androgen receptor expression is not associated with pathological stage, grade, gender or outcome in bladder cancer: A large multi-institutional study. BJU Int 108: 24-30, 2011.

12. Moher D, Liberati A, Tetzlaff J and Altman DG; PRISMA Group: Preferred reporting items for systematic reviews and meta-analyses: The PRISMA statement. Int J Surg 8: 336-341, 2010.

13. Parmar MK, Torri V and Stewart L: Extracting summary statistics to perform meta-analyses of the published literature for survival endpoints. Stat Med 17: 2815-2834, 1998.

14. Tierney JF, Stewart LA, Ghersi D, Burdett S and Sydes MR: Practical methods for incorporating summary time-to-event data into meta-analysis. Trials 8: 16, 2007.

15. Stang A: Critical evaluation of the Newcastle-Ottawa scale for the assessment of the quality of nonrandomized studies in meta-analyses. Eur J Epidemiol 25: 603-605, 2010.

16. Higgins JP, Thompson SG, Deeks JJ and Altman DG: Measuring inconsistency in meta-analyses. BMJ 327: 557-560, 2003.

17. Egger M, Davey Smith G, Schneider M and Minder C: Bias in meta-analysis detected by a simple, graphical test. BMJ 315: 629-634, 1997.

18. Begg CB and Mazumdar M: Operating characteristics of a rank correlation test for publication bias. Biometrics 50: 1088-1101, 1994.

19. Tuygun C, Kankaya D, Imamoglu A, Sertcelik A, Zengin K, Oktay M and Sertcelik N: Sex-specific hormone receptors in urothelial carcinomas of the human urinary bladder: A comparative analysis of clinicopathological features and survival outcomes according to receptor expression. Urol Oncol 29: 43-51, 2011.

20. Zheng Y, Izumi K, Yao JL and Miyamoto H: Dihydrotestosterone upregulates the expression of epidermal growth factor receptor and ERBB2 in androgen receptor-positive bladder cancer cells. Endocr Relat Cancer 18: 451-464, 2011. 
21. Miyamoto H, Yao JL, Chaux A, Zheng Y, Hsu I, Izumi K, Chang C, Messing EM, Netto GJ and Yeh S: Expression of androgen and Oestrogen receptors and its prognostic significance in urothelial neoplasm of the urinary bladder. BJU Int 109: 1716-1726, 2012.

22. Mashhadi R, Pourmand G, Kosari F, Mehrsai A, Salem S, Pourmand MR, Alatab S, Khonsari M, Heydari F, Beladi L and Alizadeh F: Role of steroid hormone receptors in formation and progression of bladder carcinoma: A case-control study. Urol J 11: 1968-1973, 2014.

23. Nam JK, Park SW, Lee SD and Chung MK: Prognostic value of sex-hormone receptor expression in non-muscle-invasive bladder cancer. Yonsei Med J 55: 1214-1221, 2014.

24. Jing Y, Cui D, Guo W, Jiang J, Jiang B, Lu Y, Zhao W, Wang X, Jiang Q, Han B and Xia S: Activated androgen receptor promotes bladder cancer metastasis via Slug mediated epithelial-mesenchymal transition. Cancer Lett 348: 135-145, 2014.

25. Williams EM, Higgins JP, Sangoi AR, McKenney JK and Troxell ML: Androgen receptor immunohistochemistry in genitourinary neoplasms. Int Urol Nephrol 47: 81-85, 2015.

26. Izumi K, Ito Y, Miyamoto H, Miyoshi Y, Ota J, Moriyama M, Murai T, Hayashi H, Inayama Y, Ohashi K, et al: Expression of androgen receptor in non-muscle-invasive bladder cancer predicts the preventive effect of androgen deprivation therapy on tumor recurrence. Oncotarget 7: 14153-14160, 2016.

27. Chang CS, Kokontis J and Liao ST: Molecular cloning of human and rat complementary DNA encoding androgen receptors. Science 240: 324-326, 1988.

28. Culig Z and Santer FR: Androgen receptor co-activators in the regulation of cellular events in prostate cancer. World J Urol 30: 297-302, 2012.
29. Lombard AP and Mudryj M: The emerging role of the androgen receptor in bladder cancer. Endocr Relat Cancer 22: R265-R277, 2015.

30. Chang C, Lee SO, Yeh S and Chang TM: Androgen receptor (AR) differential roles in hormone-related tumors including prostate, bladder, kidney, lung, breast and liver. Oncogene 33: 3225-3234, 2014

31. Guan Z, Li C, Fan J, He D and Li L: Androgen receptor (AR) signaling promotes RCC progression via increased endothelial cell proliferation and recruitment by modulating $\mathrm{AKT} \rightarrow \mathrm{NF}-\kappa \mathrm{B} \rightarrow \mathrm{CXCL5}$ signaling. Sci Rep 6: 37085, 2016.

32. Miyamoto $H$, Yang $Z$, Chen YT, Ishiguro $H$, Uemura $H$, Kubota Y, Nagashima Y, Chang YJ, Hu YC, Tsai MY, et al: Promotion of bladder cancer development and progression by androgen receptor signals. J Natl Cance Inst 99: 558-568, 2007.

33. Lopez-Beltran A and Montironi R: Non-invasive urothelial neoplasms: According to the most recent WHO classification. Eur Urol 46: 170-176, 2004.

34. Chen C, Qi XJ, Cao YW, Wang YH, Yang XC, Shao SX and Niu HT: Bladder tumor heterogeneity: The impact on clinical treatment. Urol Int 95: 1-8, 2015.

35. Witjes JA, Compérat E, Cowan NC, De Santis M, Gakis G, Lebret T, Ribal MJ, Van der Heijden AG and Sherif A; European Association of Urology: EAU guidelines on muscle-invasive and metastatic bladder cancer: Summary of the 2013 guidelines. Eur Urol 65: 778-792, 2014. 